\title{
Onset of secondary progressive phase and long-term evolution of multiple sclerosis
}

\author{
Antonio Scalfari, ${ }^{1}$ Anneke Neuhaus, ${ }^{2}$ Martin Daumer, ${ }^{2}$ Paolo Antonio Muraro, ${ }^{1}$ \\ George Cornell Ebers ${ }^{3}$
}

${ }^{1}$ Division of Experimental Medicine, Centre for Neuroscience, Imperial College London, London, UK

${ }^{2}$ Sylvia Lawry Centre, Munich, Germany

${ }^{3}$ Department of Clinical Neurology, University of Oxford, Oxford, UK

\section{Correspondence to}

Professor George Cornell Ebers, Department of Clinical Neurology, University of Oxford, Level 3, West Wing John Radcliffe Hospital, Oxford OX3 9DU, UK; george.ebers@ndcn.ox.ac.uk

Received 9 October 2012 Revised 13 February 2013 Accepted 15 February 2013 Published Online First 13 March 2013

\section{SLinked}

- http://dx.doi.org/10.1136/ jnnp-2012-304611

To cite: Scalfari $A$ Neuhaus A, Daumer M, et al. I Neurol Neurosurg Psychiatry 2014;85:67-75.

\section{ABSTRACT \\ Objectives To assess factors affecting the rate of conversion to secondary progressive (SP) multiple sclerosis (MS) and its subsequent evolution.}

Methods Among 806 patients with relapsing remitting (RR) onset MS from the London Ontario database, we used Kaplan-Meier, Cox regression and multiple logistic regression analyses to investigate the effect of baseline clinical and demographic features on (1) the probability of, and the time to, SP disease, (2) the time to bedbound status (Disability Status Scale (DSS 8)) from onset of progression.

Results The risk of entering the SP phase increased proportionally with disease duration $(O R=1.07$ for each additional year; $p<0.001)$. Shorter latency to SP was associated with shorter times to severe disability. The same association was found even when patients were grouped by number of total relapses before progression. However, the evolution of the SP phase was not influenced by the duration of the RR phase. Male sex $(H R=1.41 ; p<0.001)$, older age at onset (age $\leq 20$ and $21-30$ vs $>30 \mathrm{HR}=0.52(p<0.001), 0.65(p<0.001)$, respectively) and high early relapse frequency (1-2 attacks vs $\geq 3 \mathrm{HR}=0.63(p<0.001), 0.75(p=0.04)$, respectively) predicted significantly higher risk of SP MS and shorter latency to progression. Times to DSS 8 from onset of progression were significantly shorter among those with high early relapse frequency ( $\geq 3$ attacks), and among those presenting with cerebellar and brainstem symptoms.

Conclusions The onset of SP MS is the dominant determinant of long-term prognosis, and its prevention is the most important target measure for treatment. Baseline clinical features of early relapse frequency and age at onset can be used to select groups at higher risk of developing severe disability based on the probability of their disease becoming progressive within a defined time period.

\section{INTRODUCTION}

Most patients with multiple sclerosis (MS) during the relapsing remitting (RR) phase have only moderate disability (Disability Status Scale (DSS) ${ }^{1} \leq 3$ ), ${ }^{2-4}$ and a sizeable minority have little or none. The secondary progressive (SP) phase, which supervenes after a variable time from disease onset, ${ }^{5-7}$ leads to severe outcomes (requiring walking aids or worse), resulting from the progressive accumulation of unremitting disability. ${ }^{3}$ 8-11 Disease evolution during the progressive phase has been reported to be homogeneous among progressive subtypes 281213 and independent of factors preceding its onset. ${ }^{3} 91114$ Therefore, the largely unpredictable outcome of individual cases is mainly accounted for by the mechanism driving the evolution of the RR phase.

The conversion from RR MS to SP MS is the key determinant of prognosis and its prevention or delay is a robust marker for long-term disability and a major therapeutic target. Factors affecting the probability of entering the progressive phase should be used to improve the design of randomised controlled trials (RCTs). Predictors of SP onset have been explored by several studies, generally reporting a high proportion of censored information (patients not reaching SP) and, to some extent, contradictory results. ${ }^{6} 1^{10}{ }^{15-21}$ The London Ontario (LO) database, comprises a large number of patients attaining SP and severe disability outcome (DSS 8) over a period of 28 years, ${ }^{3}$ and thus is an ideal dataset for further assessing disease evolution before and after the onset of progression. We investigated the prognostic value of baseline clinical and demographic features for the onset and evolution of SP MS. In addition, in order to explore the potential usefulness of SP onset as primary endpoint in future RCTs, we developed models predicting the risk of becoming severely disabled based on the probability of developing a progressive course.

\section{METHODS}

The characteristics of the LO database have been extensively outlined in previous publications. ${ }^{2} 31422$ The London Multiple Sclerosis Clinic (London Health Sciences Centre, Canada), established in 1972, provides long-term care for patients with MS from south-western Ontario. Accrual ended in 1984 and patients were evaluated annually or semiannually, regardless of clinical course, over a period of 28 years (from 1972 to 2000). The shortest follow-up was 16 years. At each visit new information was collected and data previously recorded were confirmed. Disability was assessed using the DSS. ${ }^{1}$ No patient received disease-modifying therapies. The database was recently (2009) subjected to a rigorous data quality process.

\section{Definitions of outcomes}

Exacerbations were defined as acute development of new symptoms, or worsening of existing symptoms, lasting for $>24$ h. $^{5} 23$ The onset attack was counted as 'first relapse'. Progressive disease (SP MS) was defined as at least 1 year of continuous deterioration, regardless of the rate of worsening. 
Transitory plateaus and small temporary improvements in the relentlessly progressive course were recorded in the long term, although steady progression was the rule. ${ }^{2} 12$ In order to resolve ambiguities over time, documentations collected for the onset of the SP phase and for the hard disability endpoint of restriction to bed with preserved use of arms (DSS 8), which were the focus of this study, were repeatedly checked during the observation period. A minority of unrecorded DSS scores or SP onset information was derived from the description of the neurological findings only when unambiguous. Otherwise the database was left blank for that specific visit. At the end of the observation period (2000) data on SP onset were carefully reviewed before we carried out extensive analyses on the progressive phase. $^{2}$

\section{Statistical methods}

The analysis focused on the disease courses of 806 patients with initial RR MS. Kaplan-Meier analysis estimated times to development of SP MS from disease onset and, among patients with SP MS, times to DSS 8 from onset of progression in patients grouped by demographic and clinical baseline features: gender, age at disease onset, frequency of early relapses (number of attacks during the first 2 years), type (motor; sensory; cerebellar, brainstem; optic) and number $(1 ;>1)$ of neurological systems affected at onset. Grouping aimed for similar numbers of patients in each category; additional stratifications provided internal controls to confirm results. A log rank test was used to investigate differences among groups. Patients not reaching DSS 8 or not reaching SP but followed up for known periods were right censored. In the analyses of time to SP onset and time to DSS 8 from onset of SP, 37 and 60 patients, respectively, were excluded because of missing information on disease duration or on time to the endpoint. Multiple Cox proportional hazard analysis was used to investigate the risk of developing progressive MS and of reaching DSS 8 from onset of progression according to the concomitant effect of all clinical and demographic features. Proportional hazards assumption was checked by visual inspection of Schoenfeld residual plots and corresponding statistical tests. In addition, within the SP MS group, Kaplan-Meier analysis was used for assessing (1) times from disease onset and from onset of SP to DSS 8 in patients stratified by duration of the RR phase (latency to SP: short $=1-5$ years; intermediate $=6-$ 12 years; long $\geq 13$ years); grouping aimed at reaching similar numbers among groups. However, analysis was repeated with different grouping as internal control; (2) time to SP in patients stratified by number of total relapses during the RR phase (low =1-2; intermediate $=3-4$; high $\geq 5$ attacks); as internal control, we repeated the analysis in patients starting to progress at $\leq$ DSS 2, where the onset of progression is more easily detect-

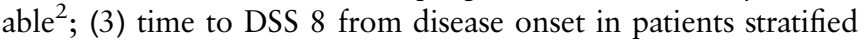
by duration of the RR phase and by number of total relapses during the RR phase. Finally, binary logistic regression analysis was used to calculate the independent predictive effect of disease duration, frequency of early relapses and age at onset on the probability of entering the SP phase, expressed by OR. We used a generalised linear model to predict the probabilities of converting to SP MS according to different values of disease duration. Multiple binary logistic regression analysis allowed us to build up models predicting the variation according to disease duration of the probability of converting to SP MS influenced by age at onset and frequency of early relapses. The $\chi^{2}$ test and the Wilcoxon signed-rank test were used for comparisons of categorical data and quantitative data, respectively.

A statistical analysis plan was set up and agreed among the authors. $^{24}$ All statistical analyses were performed using SPSS software (V.15) by one author (AS) and independently recalculated at the Sylvia Lawry Centre using R software. ${ }^{25}$

\section{Standard protocol approvals, registrations} and patient consents

Written informed consent was obtained from all patients (or guardians of patients) participating in the study (consent for research). Approval was received from an ethical standards committee for experiments using human subjects including at the LO.

\section{RESULTS}

By the end of the study, of 806 patients with an initial RR course, 534 (66.3\%) had SP MS and 272 (33.7\%) remained in the RR phase (table 1). The group who did not enter the progressive phase contained a larger percentage of women $(76.5 \%$ vs $64.8 \% ; \mathrm{p}<0.001)$, had a younger mean age at disease onset ( 26.8 vs 29.4 years; $p<0.001$ ) and shorter mean disease duration (20.3 vs 26.5 years; $\mathrm{p}<0.001)$. However, in both groups early relapse frequency ( 0.97 vs 0.91 attacks/year; $p=0.18)$ was similar and most patients presented with monosymptomatic onset, mainly characterised by sensory symptoms. Among patients with SP MS, 92\% reached DSS 6, 68\% reached DSS 8 and $23 \%$ reached DSS 10; times to endpoints were 14, 24 and 45 median years from disease onset and 3, 12 and 30 median years from onset of SP, respectively (table 1 ).

\section{Latency to progression}

Binary logistic regression analysis was used to calculate the risk of MS becoming progressive. The probability of entering the SP phase increased proportionally with disease duration (regression coefficient $=0.069 ;$ OR $=1.07$ (95\% CI 1.05 to 1.09$)$ for each additional year; $\mathrm{p}<0.001)$, becoming double $(\mathrm{OR}=1.99$, probability $=43.9 \%)$ and quadruple $(\mathrm{OR}=3.97$, probability $=61.0 \%)$ after 10 and 20 years from disease onset, respectively (figure 1). This effect was previously shown to be independent of age at disease onset. ${ }^{14}$ Of 769 patients with relapsing onset for whom information was available, Kaplan-Meier analysis estimated a mean period of 21.4 (95\% CI 19.5 to 23.1) years before to enter the SP phase. By 6 and 15 (median time) years from onset $25 \%$ and $50 \%(n=387)$, respectively, had SP MS. In the following 20 years few additional patients attained progression, to a final total of $66 \%$ (figure 2). Accordingly, the rate of conversion to SP MS was much higher during the first 15 years $(25.8$ patients/year) of disease duration than during the following 15 years (8.1 patients/year) (figure 2).

At the end of the study, of the group who remained in the RR phase, 50\% had been observed for 20 years (median disease duration). In $25 \%(n=68)$ progression had not occurred after $\geq 24$ years from onset and for some $(n=13)$ not even after $\geq 40$ years. Among patients with SP MS, latency to progression varied greatly (from 1 to 36 years), ranging from a mean of 5 years in the quickest $25 \%$ to $>15$ years in the slowest $25 \%$. The mean time to enter the progressive phase was 10.7 years (95\% CI 10.0 to 11.3 ); by 5,9 (median time) and 15 years, $25 \%, 50 \%$ and $75 \%$, of patients with RR MS had SP disease.

\section{Latency to progression and relapses}

Although a larger number of inflammatory attacks during the first 2 years (early relapses) were previously shown to predict shorter latency to progression, ${ }^{3}$ Kaplan-Meier analysis conversely demonstrated that the number of total relapses during the RR phase was associated with the time to SP onset. This was significantly $(p<0.001)$ longer among those with five or more 
Table 1 Clinical and demographic features of RR and SP patients

\begin{tabular}{|c|c|c|c|}
\hline Characteristics & RR MS & SP MS & $p$ Value \\
\hline $\mathrm{N}$ & 272 & 534 & \\
\hline Women, n (\%) & $208(76.5)$ & $346(64.8)$ & $<0.001^{*}$ \\
\hline Men, $n(\%)$ & $64(23.5)$ & $188(35.2)$ & \\
\hline F/M ratio & 3.3 & 1.8 & \\
\hline Age at onset (years), mean (SD) & $26.8(8.13)$ & $29.4(9.22)$ & $<0.001 \dagger$ \\
\hline Disease duration (years), mean (SD) & $20.3(9.18)$ & $26.5(10.0)$ & $<0.001 \dagger$ \\
\hline Monosymptomatic onset, n (\%) & $173(63.6)$ & $362(67.8)$ & $0.19^{*}$ \\
\hline Polysymptomatic onset, n (\%) & $97(35.7)$ & $165(30.9)$ & \\
\hline Information not available, n (\%) & $2(0.7)$ & $7(1.3)$ & \\
\hline \multicolumn{4}{|l|}{ Type of initial presentation, n (\%) } \\
\hline Motor & $40(14.7)$ & $105(19.7)$ & $0.08^{*}$ \\
\hline Sensory & $159(58.5)$ & $279(52.2)$ & $0.09^{*}$ \\
\hline Cerebellar & $16(5.9)$ & $35(6.6)$ & $0.71^{*}$ \\
\hline Brainstem & $59(21.7)$ & $108(20.2)$ & $0.63^{*}$ \\
\hline Optic & $56(20.6)$ & $118(22.1)$ & $0.62^{*}$ \\
\hline Bowel/bladder & $9(3.3)$ & $16(3.0)$ & $0.81^{*}$ \\
\hline Relapse rate in year 1-2 (attacks/year), mean (median) & $0.97(0.75)$ & $0.91(0.5)$ & $0.18 \dagger$ \\
\hline Relapse rate in RR phase (attacks/year), mean (median) & & $0.65(0.40)$ & \\
\hline Duration of RR phase (years), mean (median) & & $10.7(9)$ & \\
\hline DSS score at onset of SP, mean (median) & & $2.9(3)$ & \\
\hline \multicolumn{4}{|c|}{ Kaplan-Meier estimated mean (median) time (years) from disease onset to } \\
\hline DSS 6 & & $15.7(14)$ & \\
\hline DSS 8 & & $26.5(24)$ & \\
\hline DSS 10 & & $44.8(45)$ & \\
\hline \multicolumn{4}{|c|}{ Kaplan-Meier estimated mean (median) time (years) from OPP to } \\
\hline DSS 6 & & $5.3(3)$ & \\
\hline DSS 8 & & $15.2(12)$ & \\
\hline DSS 10 & & $32.5(30)$ & \\
\hline
\end{tabular}

attacks (mean 12.2 years) than among those with one to two attacks (mean 8.8 mean) (figure 3 ).

\section{Latency to progression and late outcome}

Among patients with SP MS grouped according to duration of the $\mathrm{RR}$ phase (time to SP short $=1-5$ years; intermediate $=$ 6-12 years; long $\geq 13$ years), Kaplan-Meier analysis was used to estimate the time to bedbound status (DSS 8) from disease onset and from onset of progression. A longer time to SP was associated with significantly longer time to DSS 8 from disease onset. However, the slope of the SP phase (time to DSS 8 from SP onset) was largely unaffected by the duration of the RR phase (figure 4). In addition, latency to SP had the same predictive effect among patients grouped by number of total relapses
Figure 1 Binary logistic regression analysis. Risk of converting to secondary progressive (SP) multiple sclerosis according to disease duration (regression coefficient $=0.069 ; \mathrm{OR}=1.07$ (95\% Cl 1.05 to 1.09 ) for each additional year; $p<0.001)$. Access the article online to view this figure in colour.

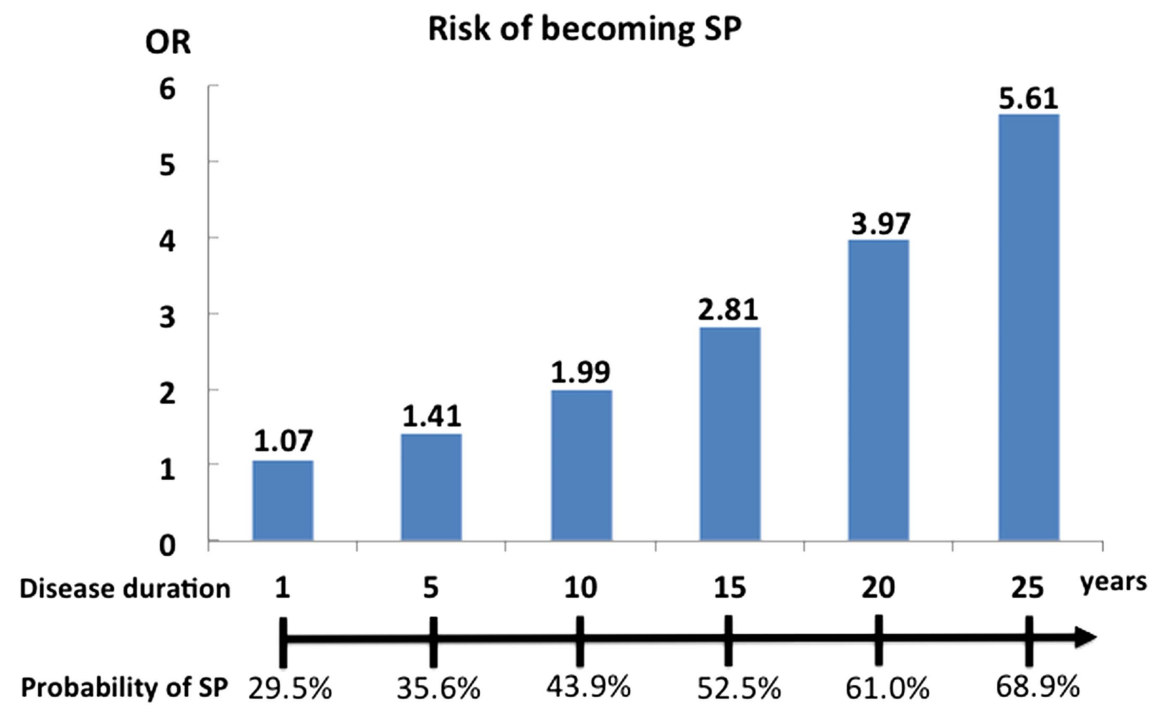




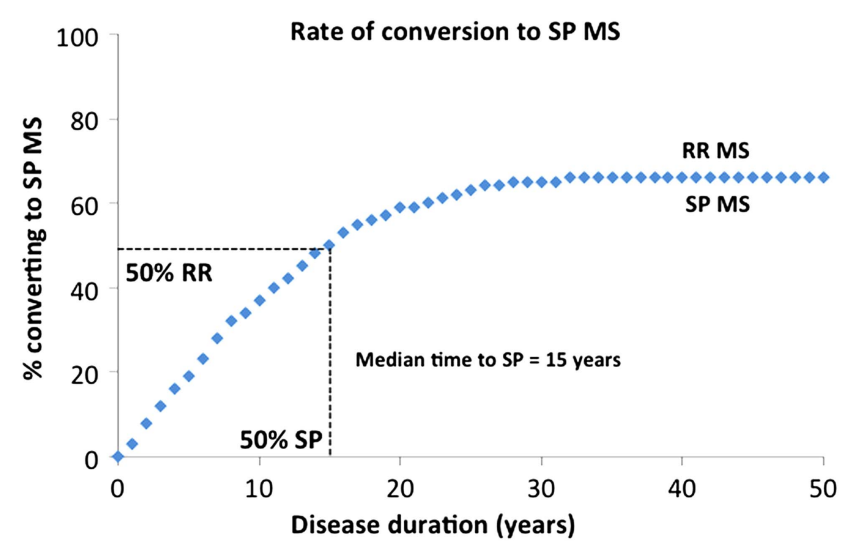

Figure 2 Kaplan-Meier analysis. Cumulative percentage of patients converting to secondary progressive multiple sclerosis (SP MS). Median time to $\mathrm{SP}=15$ years.

before progression. Shorter latency to SP was associated with significantly shorter times to DSS 8 among those with low (1$2)$, intermediate $(3-4)$ or high $(\geq 5)$ number of attacks during the RR phase (table 2).

Factors predicting the onset and the evolution of SP MS Onset of SP phase

Kaplan-Meier survival analysis demonstrated that male sex, older age at disease onset and higher early (first 2 years) relapse frequency were associated with a significantly shorter time to onset of SP (table 3). There was a mean difference of 6.5 years between men (15.9 years) and women (22.4 years) for MS becoming progressive. Similar results as previously shown, latency to progression was significantly shorter among those who were older at disease onset ${ }^{2} \begin{array}{llllll}11 & 14 & 16-19 & 26 & 27 & \text { and among }\end{array}$ those with large $(\geq 3)$ number of relapses during the first 2 years. ${ }^{3}$ Type and number of symptoms at clinical presentation did not have any significant effect on the time to SP. Multiple Cox regression analysis, assessing the concomitant predictive effect of all baseline features, confirmed a higher probability of developing SP MS in men, in those older at disease onset and in those with high early relapse frequency (table 3). The impact of these three variables was independent, as the size of the predictive effect did not change when assessed by univariate or multivariate analysis.

Models predicting risk of becoming progressive

Multiple binary logistic regression analysis allowed calculation of the probability of developing progressive disease based on the number of early relapses (regression coefficient $=0.101$; $\mathrm{OR}=1.11$ (95\% CI 0.96 to 1.27 ) for each additional relapse; $\mathrm{p}<0.001$ ) and age at disease onset (regression coefficient $=0.049$; $\mathrm{OR}=1.05 \quad(95 \%$ CI $1.02 \quad 1.07)$ for each additional year; $\mathrm{p}<0.001$ ) and its variation according to disease duration (regression coefficient $=0.074 ; \mathrm{OR}=1.08$ (95\% CI 1.05 to 1.09 ) for each additional year; $\mathrm{p}<0.001)$. Based on number of attacks during the first 2 years and age at first symptom we created models predicting the probability of entering the progressive phase and its variation over time (table 4). Two years after disease
Figure 3 Kaplan-Meier analysis. Time to secondary progressive (SP) onset in patients grouped by number of total relapses during the relapsing remitting phase: $1-2 ; 3-4 ; \geq 5$.

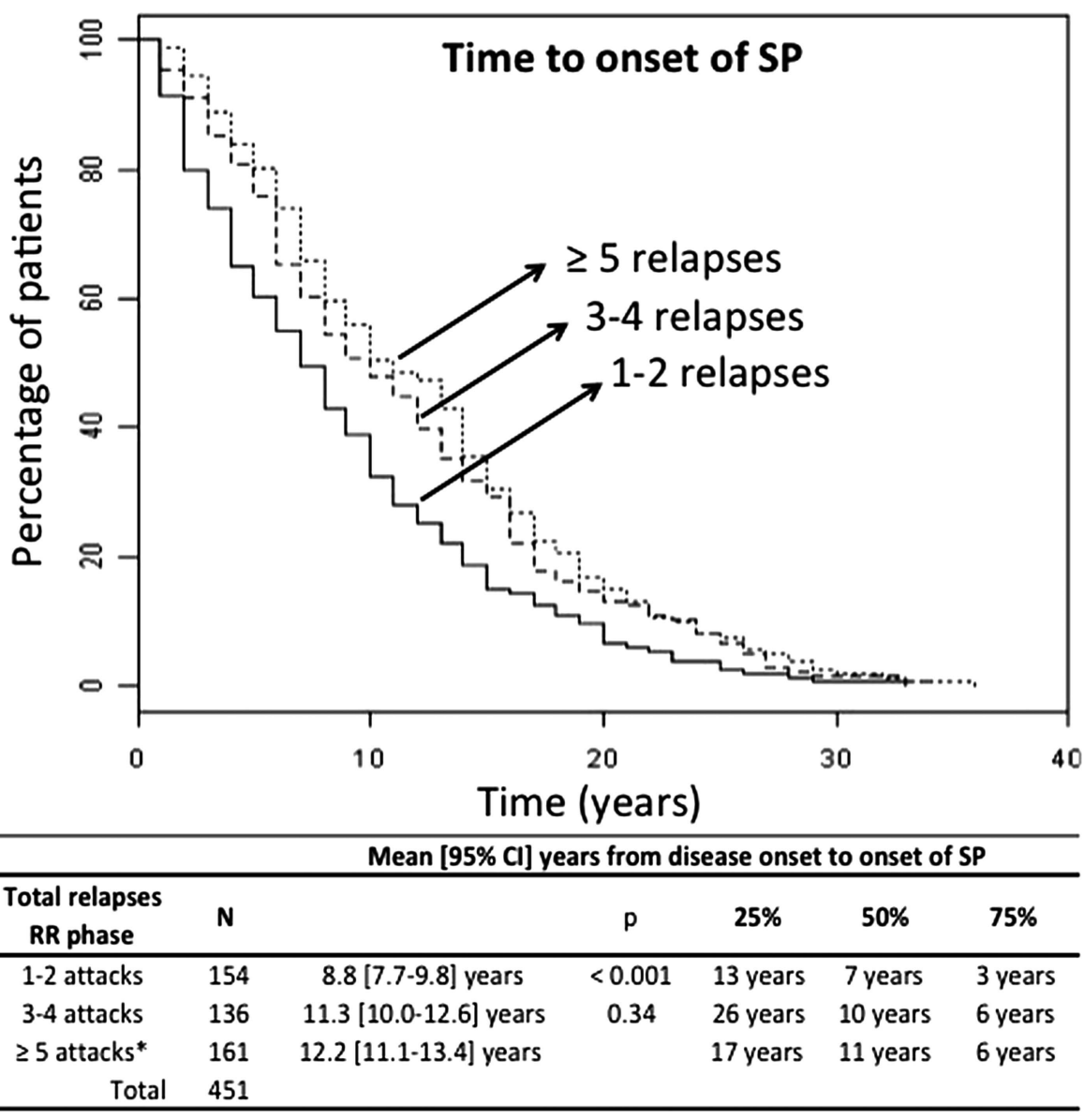

* Reference category 


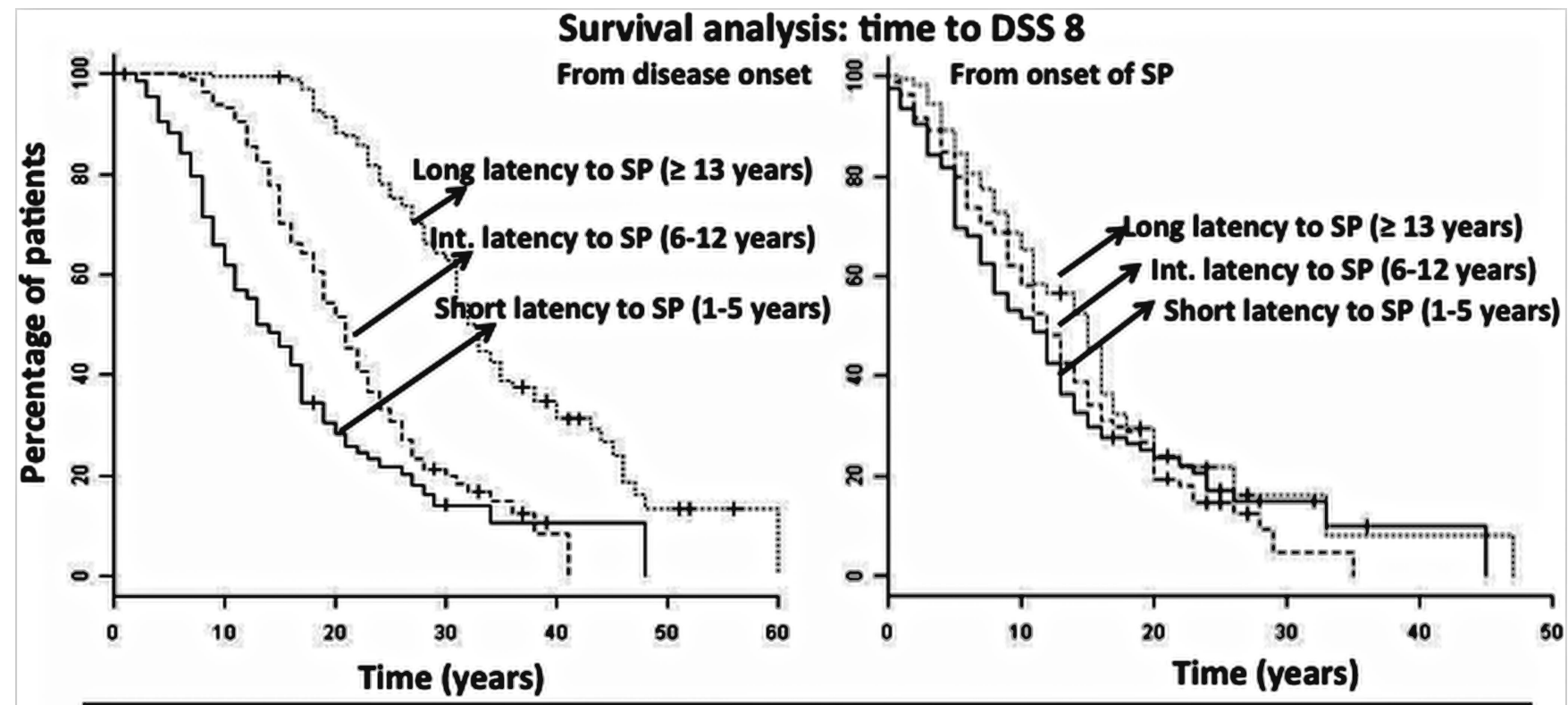

\begin{tabular}{|c|c|c|c|c|c|c|}
\hline & & \multicolumn{5}{|c|}{ Mean [95\% Cl] years from disease onset to DSS 8} \\
\hline Duration of RR phase & N (\% censored) & & $\mathbf{p}$ & $25 \%$ & $\mathbf{5 0 \%}$ & $75 \%$ \\
\hline $1-5$ years & $137(25 \%)$ & $17.5[14.8-19.9]$ years & $<0.001$ & 21 years & 14 years & 8 years \\
\hline $6-12$ years & $170(28 \%)$ & $22.2[20.6-23.8]$ years & $<0.001$ & 27 years & 21 years & 15 years \\
\hline$\geq 13$ years* & $169(45 \%)$ & $33.9[33.0-38.0]$ years & & 45 years & 33 years & 26 years \\
\hline \multirow[t]{2}{*}{ Total } & $476(33 \%)$ & & & & & \\
\hline & & \multicolumn{5}{|c|}{ Mean $[95 \% \mathrm{Cl}]$ years from onset of SP to DSS 8} \\
\hline $1-5$ years & $137(25 \%)$ & $14.4[11.9-16.9]$ years & 0.03 & 19 years & 11 years & 5 years \\
\hline $6-12$ years & $170(28 \%)$ & $13.7[12.2-15.3]$ years & 0.10 & 20 years & 12 years & 6 years \\
\hline$\geq 13$ years* & $167(45 \%)$ & $16.7[14.1-20.2]$ years & & 20 years & 15 years & 8 years \\
\hline Total & $474(33 \%)$ & & & & & \\
\hline
\end{tabular}

\section{* Reference category}

Figure 4 Kaplan-Meier analysis. Times to Disability Status Scale 8 (DSS 8) from disease onset and from onset of secondary progressive (SP) disease in patients grouped by duration of the relapsing remitting (RR) phase (1-5 years; $6-12$ years; $\geq 13$ years).

onset, three relapses resulted in ORs of $4.1,6.7$ or 10.9 in patients aged 20,30 or 40 at first symptom, respectively. The probability of developing SP MS increased with disease duration, becoming double at 10 years $(\mathrm{OR}=7.5,12.1,19.8$, respectively), approximately fivefold higher at 20 years $(\mathrm{OR}=15.6,25.5,41.4$, respectively) and sixfold higher at 25 years $(\mathrm{OR}=22.6,36.9$, 60.0 , respectively) from disease onset (table 4 ).
Evolution of SP phase

Kaplan-Meier analysis showed a mean of 15.2 (95\% CI 13.7 to 16.5 ) years for reaching DSS 8 from onset of progression. Half of the patients attained the endpoint within 12 years (median time by Kaplan-Meier estimate) from onset of SP MS. Despite their predictive effect for development of SP MS, gender and age at disease onset were found not to affect the slope of the SP

Table 2 Kaplan-Meier survival analysis

\begin{tabular}{|c|c|c|c|c|c|c|c|c|c|}
\hline \multirow[b]{3}{*}{ Duration of RR phase (years) } & \multirow[b]{3}{*}{ n (\% censored) } & \multicolumn{3}{|c|}{ Total relapses $=1-2$} & \multicolumn{3}{|c|}{ Total relapses $=3-4$} & \multicolumn{2}{|c|}{ Total relapses $\geq 5$} \\
\hline & & \multicolumn{8}{|c|}{ Mean (median) years from disease onset to DSS 8} \\
\hline & & & $\mathrm{p}$ Value & n (\% censored)) & & $\mathrm{p}$ Value & n (\% censored) & & $\mathrm{p}$ Value \\
\hline $1-5$ & $57(28)$ & $20.0(17)$ & $<0.001$ & $30(20)$ & $13.7(10)$ & $<0.001$ & $31(25)$ & $15.1(11)$ & $<0.001$ \\
\hline $6-12$ & $50(26)$ & $25.0(23)$ & $<0.001$ & $48(31)$ & $21.5(21)$ & $<0.001$ & $52(23)$ & $18.9(19)$ & $<0.001$ \\
\hline$\geq 13^{*}$ & $37(35)$ & $34.0(33)$ & & $45(40)$ & $34.2(31)$ & & $71(54)$ & $34.7(33)$ & \\
\hline Total & 144 & & & 123 & & & 154 & & \\
\hline
\end{tabular}


Table 3 Kaplan Meier survival analysis and Cox regression multiple analysis: estimated times to onset of SP and to DSS 8 from onset of progression, and estimated risk (HR) of attaining SP and DSS8 from onset of progression in patients grouped by clinical and demographic baseline features

\begin{tabular}{|c|c|c|c|c|c|c|c|c|c|c|}
\hline & \multirow[b]{3}{*}{ n (\% censored) } & \multicolumn{4}{|c|}{ Survival analysis from disease onset } & \multirow[b]{3}{*}{$\mathrm{n}$ (\% censored) } & \multicolumn{4}{|c|}{ Survival analyses from onset of progression (SP patients only) } \\
\hline & & \multicolumn{2}{|l|}{ Kaplan-Meier analysis } & \multicolumn{2}{|c|}{ Cox regression multiple analysis } & & \multicolumn{2}{|c|}{ Kaplan-Meier analysis } & \multicolumn{2}{|c|}{ Cox regression multiple analysis } \\
\hline & & $\begin{array}{l}\text { Mean years to onset } \\
\text { of SP }(95 \% \mathrm{Cl})\end{array}$ & $\mathrm{p}$ Value & $\begin{array}{l}\text { Risk of converting } \\
\text { to SP MS; HR }(95 \% \mathrm{Cl})\end{array}$ & $\mathrm{p}$ Value & & $\begin{array}{l}\text { Mean years to } \\
\text { DSS } 8(95 \% \mathrm{Cl})\end{array}$ & $\mathrm{p}$ Value & $\begin{array}{l}\text { Risk of reaching } \\
\text { DSS 8; HR }(95 \% \mathrm{CI})\end{array}$ & $\mathrm{p}$ Value \\
\hline Total population & $769(33.8)$ & $21.4(19.5$ to 23.1$)$ & & & & $474(33.1)$ & $15.2(13.7$ to 16.5$)$ & & & \\
\hline \multicolumn{11}{|l|}{ Gender } \\
\hline Male & $240(24.6)$ & $15.9(13.4$ to 17.1$)$ & $<0.001$ & 1.41 (1.17 to 1.71$)$ & 0.001 & $165(33.9)$ & $15.5(12.8$ to 16.0$)$ & 0.92 & $1.03(0.81$ to 1.31$)$ & 0.80 \\
\hline Female* & $529(38.0)$ & $22.4(21.4$ to 26.1$)$ & & & & $309(32.7)$ & $14.7(13.3$ to 16.7$)$ & & & \\
\hline \multicolumn{11}{|l|}{ Age at onset } \\
\hline$\leq 20$ & $145(41.1)$ & $25.8(21.7$ to 29.9$)$ & $<0.001$ & $0.52(0.40$ to 0.68$)$ & $<0.001$ & $80(35.0)$ & $13.9(10.2$ to 17.7$)$ & 0.11 & 1.55 (1.10 to 2.18$)$ & 0.01 \\
\hline $21-30$ & $371(38.1)$ & 20.2 (18.2 to 21.7$)$ & $<0.001$ & 0.65 (0.53 to 0.80$)$ & $<0.001$ & $207(30.0)$ & $15.3(13.3$ to 17.4$)$ & 0.44 & 1.15 (0.89 to 1.48$)$ & 0.29 \\
\hline$>30^{*}$ & $285(24.6)$ & $15.3(13.3$ to 17.3$)$ & & & & $187(35.8)$ & $15.0(13.4$ to 16.6$)$ & & & \\
\hline \multicolumn{11}{|c|}{ Relapses during year $1-2$} \\
\hline 1 & $380(28.7)$ & 19.9 (18.3 to 21.5$)$ & 0.01 & $0.63(0.49$ to 0.80$)$ & $<0.001$ & $246(34.1)$ & $16.4(14.5$ to 18.2$)$ & $<0.001$ & $0.47(0.35$ to 0.63$)$ & $<0.001$ \\
\hline 2 & $179(30.2)$ & 16.7 (14.6 to 18.9$)$ & 0.38 & 0.75 (0.57 to 0.99$)$ & 0.041 & $121(38.8)$ & $14.2(12.5$ to 15.9$)$ & $<0.001$ & 0.53 (0.38 to 0.74$)$ & $<0.001$ \\
\hline$\geq 3^{*}$ & $149(34.2)$ & 15.1 (12.8 to 17.4$)$ & & & & $93(24.7)$ & $9.6(8.2$ to 11.1$)$ & & & \\
\hline \multicolumn{11}{|c|}{ No of symptoms at disease onset } \\
\hline 1 & $515(32.6)$ & 20.3 (19.2 to 23.6$)$ & 0.75 & 1.19 (0.92 to 1.54$)$ & 0.17 & $321(35.2)$ & 15.1 (13.7 to 16.3$)$ & 0.17 & $1.00(0.72$ to 1.40$)$ & 0.98 \\
\hline$>1^{*}$ & $245(36.7)$ & 19.9 (16.6 to 21.1$)$ & & & & $146(28.1)$ & 13.9 (11.9 to 16.7$)$ & & & \\
\hline \multicolumn{11}{|c|}{ Type of symptoms at disease onset } \\
\hline \multicolumn{11}{|c|}{ Motor } \\
\hline Present & $139(28.8)$ & 18.0 (14.1 to 18.7$)$ & 0.18 & $1.22(0.93$ to 1.61$)$ & 0.15 & $89(28.1)$ & 15.6 (12.5 to 18.7$)$ & 0.87 & $1.12(0.78$ to 1.61$)$ & 0.55 \\
\hline Absent ${ }^{*}$ & $630(34.9)$ & 20.0 (19.7 to 23.8$)$ & & & & $385(34.3)$ & 15.1 (13.5 to 16.7$)$ & & & \\
\hline \multicolumn{11}{|l|}{ Sensory } \\
\hline Present & $419(35.6)$ & 20.7 (17.7 to 21.2$)$ & 0.41 & $0.99(0.75$ to 1.30$)$ & 0.93 & $255(32.9)$ & 15.3 (13.8 to 17.6$)$ & 0.36 & 1.01 (0.71 to 1.41$)$ & 0.97 \\
\hline Absent* & $350(31.7)$ & 19.7 (18.2 to 23.4$)$ & & & & $219(33.3)$ & $13.9(12.3$ to 15.4$)$ & & & \\
\hline \multicolumn{11}{|l|}{ Cerebellar } \\
\hline Present & $46(30.4)$ & 18.7 (12.9 to 20.9$)$ & 0.62 & $1.16(0.79$ to 1.71$)$ & 0.44 & $30(16.7)$ & 9.5 (6.1 to 11.3$)$ & 0.001 & 2.21 (1.40 to 3.37$)$ & $<0.001$ \\
\hline Absent* & $723(34.0)$ & 19.7 (19.5 to 23.3$)$ & & & & $444(34.2)$ & $14.8(14.0$ to 17.0$)$ & & & \\
\hline \multicolumn{11}{|l|}{ Brainstem } \\
\hline Present & $154(36.4)$ & 19.2 (16.3 to 24.3$)$ & 0.75 & $1.12(0.83$ to 1.52$)$ & 0.44 & $91(23.1)$ & 11.0 (8.8 to 13.0 ) & $<0.001$ & 1.91 (1.32 to 2.79 ) & $<0.001$ \\
\hline Absent ${ }^{*}$ & $615(33.2)$ & 20.1 (17.4 to 19.8$)$ & & & & $383(35.5)$ & $15.7(! 4.5$ to 17.8$)$ & & & \\
\hline \multicolumn{11}{|l|}{ Optic } \\
\hline Present & $170(32.4)$ & 20.3 (16.2 to 20.7$)$ & 0.86 & $1.04(0.78$ to 1.39$)$ & 0.77 & $110(38.2)$ & 14.9 (12.9 to 16.7$)$ & 0.22 & $0.90(0.62$ to 1.30$)$ & 0.58 \\
\hline Absent* & $599(34.2)$ & 19.5 (19.0 to 23.0$)$ & & & & $364(31.6)$ & 14.4 (13.3 to 16.4$)$ & & & \\
\hline
\end{tabular}

Multiple Cox regression analysis: risk (HR) of conversion to SP MS and of attaining DSS 8 from onset of secondary progression according to the concomitant effect of clinical and demographic baseline features.

*Reference category.

DSS, Disability Status Scale; MS, multiple sclerosis; SP, secondary progressive. 
phase (table 3). As previously shown, high early relapse frequency predicted faster evolution of the progressive phase. ${ }^{3}$ In addition, brainstem and cerebellar presentation were associated with significantly faster attainment of DSS 8 from onset of progression (table 3). These results were confirmed by Cox regression multiple analysis, which additionally showed a slightly higher risk $(\mathrm{HR}=1.55)$ of becoming bedbound from onset of progression among those with early disease onset ( $\leq 20$ years).

\section{DISCUSSION}

Prognosis of MS has been puzzling clinicians for several decades and remains largely unpredictable for individuals. The evolution of the progressive phase has been reported to be similar among progressive subtypes ${ }^{2} \quad 8 \quad 12 \quad 13$ and unaffected by overlapping relapses $^{12} 28$ or by age at onset of progression ${ }^{2} 1422$ or by factors preceding its onset. ${ }^{3} 91114$ Long-term outcome is therefore largely determined by mechanisms associated with SP onset and driving the evolution of the RR phase, providing the likely window of therapeutic opportunity, probably in its early stages. ${ }^{3}$ The LO database, with the advantage of 28 years of follow up and with more than $65 \%$ of patients entering the progressive phase, allowed us to assess in detail disease evolution before and after the onset of progression. Data on the onset of SP have been extensively reviewed, ${ }^{2}$ providing further strength to our analyses.

We confirmed that the rate of conversion to SP MS increases proportionally to disease duration. The probability of experiencing a progressive course becomes roughly 9\% higher every 5 years, quadrupling $(\mathrm{OR}=3.97) 20$ years after onset (figure 1$)$. At the end of the observation period, about one-third $(n=272)$ of patients with RR onset did not enter the progressive phase (figure 2), despite their median disease duration of 20 years (table 1). Although we presume that most of the patients eventually developed SP MS, a minority $(n=13 ; 5 \%$ of RR patients) did not experience progression even after $\geq 40$ years of disease. This coheres with the recently reported proportion (5\%) of 'patients with benign MS, free from SP after 50 years of follow up'. ${ }^{29}$

Among those who entered the progressive phase, time to SP varied widely (from 1 to 36 years, mean time $=10.7$ years $(95 \%$ CI 10.0 to 11.3$)$ ); $25 \%$ became progressive by 5 years and at the opposite extreme $25 \%$ still had not developed an SP course by 15 years. The conversion to SP MS is the watershed event differentiating disease severity, and latency to SP accounts for the large variation of the outcome. Shorter duration of the RR phase was associated with significantly shorter times to severe disability from disease onset. However, latency to SP did not affect the evolution of the SP phase (time to severe disability from SP onset) (figure 4), confirming the amnestic nature of disease evolution, ${ }^{3}{ }^{11}$ characterised by two independent stages. Interestingly, a shorter latency to progression was associated with faster disease progression from disease onset even when patients were grouped by number of total relapses during the RR phase (table 2). This suggests a dissociation between mechanisms underlying inflammatory attacks and those leading to severe disability.

Few variables were found to reliably predict latency to progression and slope of the SP phase (table 3). As seen for late disability, ${ }^{2} 317$ but not in agreement with some previous studies, ${ }^{101518}$ type and number of neurological systems involved at onset were found not to affect the probability of MS becoming progressive. However, we confirmed that male sex, ${ }^{6} 101518$

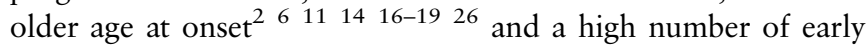
relapses 3102021 were associated with a higher probability of progressive disease and with shorter times to SP. Among the predictors of the evolution of the RR phase of MS only the frequency of early relapses affected the evolution of the SP phase. Interestingly, although type of clinical onset did not influence the latency to progression, those presenting with cerebellar and brainstem symptoms had faster evolution of the SP phase. This might suggest that when degeneration is more severe brainstem and cerebellar involvement are more likely to emerge.

The relationship between inflammatory attacks and the mechanism driving the evolution of the RR phase and the transition to SP MS has been long debated ${ }^{30-35}$ and is a crucial aspect of the disease. The annual relapse rate is commonly used as primary endpoint in RCTs and some studies have supported the use of relapse numbers as surrogates for disease progression in the short term. ${ }^{36}$ We previously reported that early relapses (during the first 2 years) are predictors of late outcomes, whereas late relapses (from year 3 up to SP onset) seemed not to affect prognosis. ${ }^{3}$ Although it is difficult to provide a biological explanation of this discrepancy, it seems that after year 2 a reversal takes place and late relapses counterbalance the effect of early relapses. ${ }^{3}$ Indeed, patients grouped by total number of relapses during the RR phase, which combines the effect of early and late relapses, were shown to reach severe disability endpoints in equal times. ${ }^{3}$ Here we further showed that time to SP onset conversely relates to the total number of attacks, being significantly shorter (3.4 mean years difference) among those with low frequency (1-2 relapses) compared with those with a high frequency of attacks ( $\geq 5$ relapses) (figure 3 ). This is in line

Table 4 Multiple binary logistic regression analysis

\begin{tabular}{|c|c|c|c|c|c|c|c|c|c|c|}
\hline & \multicolumn{5}{|c|}{ Age at disease onset } & \multicolumn{5}{|c|}{ Age at disease onset } \\
\hline & 20 years & 25 years & 30 years & 35 years & 40 years & 20 years & 25 years & 30 years & 35 years & 40 years \\
\hline \multicolumn{11}{|l|}{ REL Y1-2 } \\
\hline 1 attack & 3.3 & 4.3 & 5.5 & 7.0 & 8.9 & 6.1 & 7.8 & 10.0 & 12.6 & 16.1 \\
\hline 2 attacks & 3.7 & 4.7 & 6.0 & 7.7 & 9.9 & 6.8 & 8.6 & 11.0 & 14.0 & 17.9 \\
\hline \multirow[t]{2}{*}{3 attacks } & 4.1 & 5.2 & 6.7 & 8.6 & 10.9 & 7.5 & 9.6 & 12.1 & 15.5 & 19.8 \\
\hline & \multicolumn{5}{|c|}{ Disease duration $=2$ years } & \multicolumn{5}{|c|}{ Disease duration $=10$ years } \\
\hline 1 attack & 8.9 & 11.2 & 14.4 & 18.3 & 23.4 & 12.8 & 16.3 & 20.8 & 26.5 & 33.8 \\
\hline 2 attacks & 9.8 & 12.4 & 15.9 & 20.3 & 25.9 & 14.1 & 18.0 & 23.0 & 29.3 & 37.4 \\
\hline \multirow[t]{2}{*}{3 attacks } & 10.8 & 13.8 & 17.6 & 22.4 & 28.6 & 15.6 & 20.0 & 25.5 & 32.5 & 41.4 \\
\hline & \multicolumn{5}{|c|}{ Disease duration $=15$ years } & \multicolumn{5}{|c|}{ Disease duration $=20$ years } \\
\hline
\end{tabular}

Table shows OR expressing the probability of converting to SP MS according to concomitant effect of age at onset, number of early (during the first 2 years) relapses and disease duration. 
with a previously described shorter duration of the RR phase among single attack progressive patients (SAP) compared with patients with SP MS. ${ }^{2}$ The prognostic value of relapses appears therefore limited to early inflammatory attacks, predicting faster conversion to SP MS, ${ }^{3} 102021$ as here confirmed. However, the question of whether early relapses cause severe disability occurring 15-20 years later remains largely unanswered.

To further elucidate this point, we recently showed that $>30 \%$ of patients with three or more attacks during the first 2 years had not progressed to SP MS at the end of the observation period, discouraging any finding of a causal relationship between early attacks and late outcome. ${ }^{38}$ Overall, these results do not confirm the widespread belief that conversion to SP MS results from cumulative inflammatory attacks over time, further invalidating relapses as a surrogate marker for late disability. Indeed, therapeutic relapse suppression was shown not to affect the probability of developing severe disability in the long term. ${ }^{39}$ Furthermore, a recent study from British Columbia showed no differences in reaching the expanded DSS 6 between patients receiving inteferon therapy and two natural history cohorts. ${ }^{40}$

We acknowledge the limitation of natural history studies, which assess relapses numerically and not qualitatively. Nevertheless, the type of initial attack has been shown to have limited prognostic value, ${ }^{2} 317$ as confirmed in this study, despite speculation that severe symptoms during inflammatory attacks might impact adversely on prognosis. ${ }^{41}{ }^{42}$ However, unlike neuromyelitis optica, ${ }^{43}$ relapses in MS only rarely cause permanent severe disability, as result of incomplete recovery. Indeed, most patients in the LO database were scored at DSS $\leq 3$ when converting to SP MS, ${ }^{3}$ indicating that they had only mild disability during the RR phase. In an extensive study, following up 1078 patients for 15 years and collecting data on 2578 relapses, only a tiny proportion $(n=7)$ of attacks was shown to cause severe disability (expanded DSS $\geq 6$, sustained for at least 6 months). ${ }^{4}$

It has been debated that relapses are only the 'filtered expression' of an underlying, constantly active inflammatory process. Indeed, MRI can detect new inflammatory activity 5-10 times more than clinically evident attacks. ${ }^{44}$ However, MRI measures of inflammation have limited predictive value in the long term. The number and volume of initial T2 lesions have been shown to predict conversion to clinically definite MS but late disability only modestly, ${ }^{45-47}$ discouraging the use of MRI inflammatory markers as a surrogate for long-term disease evolution. ${ }^{48}$

Predictive models based exclusively on relapse number are not sufficiently reliable for detecting a potential effect of treatment on late outcomes. The onset of the SP phase is a robust marker for late disability and its prevention/delay should be the target of future treatments with potential neuroprotective effect. Therefore, improvements in detecting the onset of SP deserve special attention in future studies and factors predicting conversion to SP MS should be used for improving the design of RCTs. Based on the probability of experiencing an SP course, groups at higher risk of developing disability should be identified for assessing the risk/benefit ratio of more aggressive treatments. Here we used multiple binary logistic models to calculate the variation in the risk of the disease becoming progressive from hypothetical baseline situations in which patients were randomised according to early relapse number and age at onset (table 4). Given the strong influence of age on the wide variation of the latency to progression, patients with broad age ranges (eg, 18-55 years) commonly recruited in clinical trials should be restricted as they may add unexpected variation, potentially weakening randomisation schemes. In addition, high early relapse frequency, shown to be associated with higher probability of the disease becoming progressive, could be used for randomising patients destined to have a more severe disease course and therefore requiring more aggressive treatment. Finally, the rate of conversion to SP MS increases proportionally with disease duration, which should therefore be taken into consideration when randomising patients.

Contributions AS: design of the study, analysis and interpretation of the data, study supervision, drafting and revising the manuscript for intellectual content. AN, MD: design of the study, analysis and interpretation of the data. PAM: design of the study, drafting and revising the manuscript for intellectual content. GCE: design of the study, acquisition of the data, analysis and interpretation of the data, study supervision, drafting and revising the manuscript for intellectual content.

Funding This work was supported by the Italian Multiple Sclerosis Foundation (FISM; ref. 2008/R/16) (AS, PM), the Medical Research Council (PM) (ref. G0800679), The MS Society of Canada (GE), the UK MS Society (GE), the German Ministry for Education and Research (BMBF, 01GI0904, 01GI0920) (AN, MD).

Competing interests None.

Ethics approval Ethical standards committee at London Ontario Canada.

Provenance and peer review Not commissioned; externally peer reviewed.

\section{REFERENCES}

1 Kurtzke JF. A new scale for evaluating disability in multiple sclerosis. Neurology 1955;5:580-3.

2 Kremenchutzky M, Rice GP, Baskerville J, et al. The natural history of multiple sclerosis: a geographically based study 9: observations on the progressive phase of the disease. Brain 2006;129:584-94.

3 Scalfari A, Neuhaus A, Degenhardt A, et al. The natural history of multiple sclerosis: a geographically based study 10: relapses and long-term disability. Brain 2010;133:1914-29.

4 Bejaoui K, Rolak LA. What is the risk of permanent disability from a multiple sclerosis relapse? Neurology 2010;74:900-2.

5 Lublin FD, Reingold SC. Defining the clinical course of multiple sclerosis: results of an international survey. National Multiple Sclerosis Society (USA) Advisory Committee on Clinical Trials of New Agents in Multiple Sclerosis. Neurology 1996;46:907-11.

6 Vukusic S, Confavreux C. Prognostic factors for progression of disability in the secondary progressive phase of multiple sclerosis. J Neurol Sci 2003;206:135-7.

7 Weinshenker BG, Bass B, Rice GP, et al. The natural history of multiple sclerosis: a geographically based study. I. Clinical course and disability. Brain 1989;112(Pt 1): $133-46$.

8 Tremlett $\mathrm{H}$, Zhao $\mathrm{Y}$, Devonshire V. Natural history comparisons of primary and secondary progressive multiple sclerosis reveals differences and similarities. J Neurol 2009;256:374-81.

9 Leray $E$, Yaouanq J, Le Page $E$, et al. Evidence for a two-stage disability progression in multiple sclerosis. Brain 2010;133:1900-13.

10 Eriksson $\mathrm{M}$, Andersen 0 , Runmarker B. Long-term follow up of patients with clinically isolated syndromes, relapsing-remitting and secondary progressive multiple sclerosis. Mult Scler 2003:9:260-74

11 Confavreux C, Vukusic S, Adeleine P. Early clinical predictors and progression of irreversible disability in multiple sclerosis: an amnesic process. Brain 2003;126:770-82.

12 Kremenchutzky M, Cottrell $D$, Rice $G$, et al. The natural history of multiple sclerosis: a geographically based study. 7. Progressive-relapsing and relapsing-progressive multiple sclerosis: a re-evaluation. Brain 1999;122(Pt 10):1941-50.

13 Confavreux C, Vukusic S. Natural history of multiple sclerosis: a unifying concept. Brain 2006;129:606-16.

14 Scalfari A, Neuhaus A, Daumer $M$, et al. Age and disability accumulation in multiple sclerosis. Neurology 2011;77:1246-52.

15 Koch M, Kingwell E, Rieckmann P, et al. The natural history of secondary progressive multiple sclerosis. J Neurol NeurosurgPsychiatry 2010;81:1039-43.

16 Stankoff B, Mrejen S, Tourbah A, et al. Age at onset determines the occurrence of the progressive phase of multiple sclerosis. Neurology 2007;68:779-81.

17 Koch M, Mostert J, Heersema D, et al. Progression in multiple sclerosis: further evidence of an age dependent process. J Neurol Sci 2007;255:35-41.

18 Bergamaschi R, Berzuini C, Romani A, et al. Predicting secondary progression in relapsing-remitting multiple sclerosis: a Bayesian analysis. J Neurol Sci 2001;189:13-21.

19 Trojano M, Avolio C, Manzari C, et al. Multivariate analysis of predictive factors of multiple sclerosis course with a validated method to assess clinical events. J Neurol Neurosurg Psychiatry 1995;58:300-6.

20 Tremlett $H$, Yousefi M, Devonshire $V$, et al. Impact of multiple sclerosis relapses on progression diminishes with time. Neurology 2009;73:1616-23.

21 Debouverie M, Pittion-Vouyovitch S, Louis $S$, et al. Natural history of multiple sclerosis in a population-based cohort. Eur I Neurol 2008:15:916-21. 
22 Cottrell DA, Kremenchutzky M, Rice GP, et al. The natural history of multiple sclerosis: a geographically based study. 5 . The clinical features and natural history of primary progressive multiple sclerosis. Brain 1999;122(Pt 4):625-39.

23 Poser CM, Paty DW, Scheinberg L, et al. New diagnostic criteria for multiple sclerosis: guidelines for research protocols. Ann Neurol 1983;13:227-31.

24 Daumer M, Held U, Ickstadt K, et al. Reducing the probability of false positive research findings by pre-publication validation-experience with a large multiple sclerosis database. BMC Med Res Methodol 2008;8:18.

25 R.D.C. T. R: a language and enviroment for statistical computing. R Foundation for Statistical Computing, Vienna (Austria) 2008;ISBN 3-900051-07-0.

26 Confavreux C, Vukusic S. Age at disability milestones in multiple sclerosis. Brain 2006:129:595-605.

27 Tremlett H, Yinshan Z, Devonshire V. Natural history of secondary-progressive multiple sclerosis. Mult Scler 2008;14:314-24.

28 Confavreux C, Vukusic S, Moreau T, et al. Relapses and progression of disability in multiple sclerosis. N Engl J Med 2000;343:1430-8.

29 Skoog B, Runmarker B, Winblad S, et al. A representative cohort of patients with non-progressive multiple sclerosis at the age of normal life expectancy. Brain 2012;135:900-11.

30 Bjartmar C, Kinkel RP, Kidd G, et al. Axonal loss in normal-appearing white matter in a patient with acute MS. Neurology 2001;57:1248-52.

31 Prineas JW, Kwon EE, Cho ES, et al. Immunopathology of secondary-progressive multiple sclerosis. Ann Neurol 2001:50:646-57.

32 Magliozzi R, Howell O, Vora A, et al. Meningeal B-cell follicles in secondary progressive multiple sclerosis associate with early onset of disease and severe cortical pathology. Brain 2007;130:1089-104.

33 DeLuca GC, Williams K, Evangelou N, et al. The contribution of demyelination to axonal loss in multiple sclerosis. Brain 2006;129:1507-16.

34 Frischer JM, Bramow S, Dal-Bianco $A$, et al. The relation between inflammation and neurodegeneration in multiple sclerosis brains. Brain 2009:132:1175-89.

35 Trapp BD, Nave KA. Multiple sclerosis: an immune or neurodegenerative disorder? Annu Rev Neurosci 2008:31:247-69.
36 Sormani MP, Bonzano L, Roccatagliata L, et al. Surrogate endpoints for EDSS worsening in multiple sclerosis. A meta-analytic approach. Neurology 2010;75:302-9.

37 Sormani MP, Li DK, Bruzzi P, et al. Combined MRI lesions and relapses as a surrogate for disability in multiple sclerosis. Neurology 2011;77:

1684-90

38 Scalfari A, Neuhaus A, Daumer $M$, et al. Early relapses, onset of progression and late outcome in multiple sclerosis. JAMA Neurol 2013:70:214-22.

39 Ebers GC, Reder AT, Traboulsee A, et al. Long-term follow-up of the original interferon-beta1b trial in multiple sclerosis: design and lessons from a 16-year observational study. Clin Ther 2009;31:1724-36.

40 Shirani A, Zhao Y, Karim ME, et al. Association between use of interferon beta and progression of disability in patients with relapsing-remitting multiple sclerosis. JAMA 2012:308:247-56.

41 Edan $\mathrm{G}$. There is no such thing as a mild MS relapse. The mild relapse is an Anglo-Saxon delusion-Yes. Mult Scler 2012;18:927-9.

42 Lublin FD. Relapses do not matter in relation to long-term disability: no (they do). Mult Scler 2011;17:1415-16.

43 Wingerchuk DM, Lennon VA, Lucchinetti CF, et al. The spectrum of neuromyelitis optica. Lancet Neurol 2007;6:805-15.

44 McDonald WI, Miller DH, Thompson AJ. Are magnetic resonance findings predictive of clinical outcome in therapeutic trials in multiple sclerosis? The dilemma of interferon-beta. Ann Neurol 1994:36:14-18.

45 Optic Neuritis Study Group. Multiple sclerosis risk after optic neuritis: final optic neuritis treatment trial follow-up. Arch Neurol 2008:65:727-32.

46 Fisniku LK, Brex PA, Altmann DR, et al. Disability and T2 MRI lesions: a 20-year follow-up of patients with relapse onset of multiple sclerosis. Brain 2008:131:808-17.

47 Fisniku LK, Chard DT, Jackson JS, et al. Gray matter atrophy is related to long-term disability in multiple sclerosis. Ann Neurol 2008:64:247-54.

48 Daumer M, Neuhaus A, Morrissey S, et al. MRI as an outcome in multiple sclerosis clinical trials. Neurology 2009:72:705-11. 